\title{
On the local equivalence of complete bipartite and repeater graph states
}

\author{
Ilan Tzitrin* \\ Department of Physics, University of Toronto, Toronto, Ontario, M5S 1A7
}

\begin{abstract}
Classifying locally equivalent graph states, and stabilizer states more broadly, is a significant problem in the theories of quantum information and multipartite entanglement. A special focus is given to those graph states for which equivalence through local unitaries implies equivalence through local Clifford unitaries ( $\mathrm{LU} \Leftrightarrow \mathrm{LC}$ ). Identification of locally equivalent states in this class is facilitated by a convenient transformation rule on the underlying graphs and an efficient algorithm. Here we investigate the question of local equivalence of the graph states behind the all-photonic quantum repeater. We show that complete bipartite graph (biclique) states, imperfect repeater graph states and small "crazy graph" states satisfy LU $\Leftrightarrow$ LC. We continue by discussing biclique states more generally and placing them in the context of counterexamples to the LU-LC Conjecture. To this end, we offer some alternative proofs and clarifications on existing results.
\end{abstract}

\section{INTRODUCTION}

Graph states are a subset of multipartite entangled states used pervasively in quantum information protocols: quantum error-correcting codes, measurementbased quantum computing, entanglement purification, information splitting, quantum cryptography, and allphotonic quantum repeaters, among others [1-14. To define a graph state one starts by specifying the underlying (undirected) graph, $G$, through a set of vertices, $V$, and a collection of edges, $E$. Vertices are then associated with qubits and edges with entangling gates:

$$
|G\rangle \equiv \prod_{\{a, b\} \in E} \mathrm{CZ}^{a b}|+\rangle^{V},
$$

where $\mathrm{CZ}^{a b}$ is the controlled phase gate with control qubit $a$ and target qubit $b,|+\rangle \equiv \frac{1}{\sqrt{2}}(|0\rangle+|1\rangle)$, and we use the notation $|\psi\rangle^{V} \equiv \bigotimes_{a \in V}|\psi\rangle^{a}[3$. The value of graph states lies in the ability of local measurements to transform them in specified ways. In fact, any two qubits in a connected graph state can be projected to a Bell pair through a sequence of Pauli $X$ and $Z$ measurements, in what is termed localizable entanglement [3].

A study of the properties of graph states is an active area of research made difficult by their size and complexity. As the number of qubits increases, determining equivalence classes of graph states under local unitaries (LUs) or invertible stochastic local operations and classical communication (SLOCC) quickly becomes intractable 15. 16 .

On the other hand, because graph states are tied to the stabilizer formalism, as defined below, there are advantages in looking at a smaller class of operations, the local Clifford unitaries (LCs), which map the group of Pauli operators to itself under conjugation. For one, an LC operation effects a simple graphical transformation, the local complementation, on the underlying graph, as

\footnotetext{
* itzitrin@physics.utoronto.ca
}



Figure 1: Applying a local complementation to the red (lighter) vertex in either graph produces the other graph. The effect of a local complementation on a vertex is to remove the existing edges among its neighbours and add the missing ones. In light of the LC

Rule in Section IIB, the letters LC in the figure can refer both to a local complementation and to an appropriate local Clifford gate.

shown in Fig. 1 17. Furthermore, there is a classical polynomial-time algorithm that decides whether two given graphs are related by a sequence of local complementations [18, 19].

Initially, it was conjectured that two graph states equivalent under local unitaries are also equivalent under local Clifford gates, that is, $\mathrm{LU} \Leftrightarrow \mathrm{LC}[17,20]$. One direction of the conjecture - that LC equivalence implies LU equivalence - is trivial, since local Clifford gates are local unitaries themselves. The other direction, however, is false, with the smallest counterexample known at 27 qubits [21, 22].

In this paper, we seek to modestly expand the class of states for which the status of $\mathrm{LU} \Leftrightarrow \mathrm{LC}$ is known. After overviewing the stabilizer formalism and some important existing results on the local equivalence of graph states in Section II. we use Section III A to present our findings on the repeater graph states displayed in Fig. 2. These are the photonic states used in [14] to eschew quantum memories in traditional matter-based quantum repeaters. Section IIIB is reserved for a broader discussion of biclique states (see Fig. 4b), which appear in protocols beyond all-photonic repeaters [8, 12, 13, 23. Finally, in, Section IIIC, we clarify the result on counterexamples to the LU-LC Conjecture by Ji et al. [21] and place it in the context of bipartite graphs. 


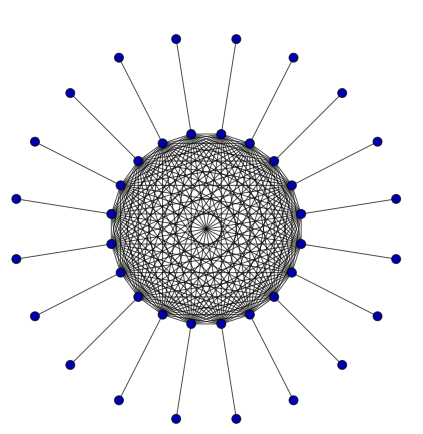

(a)

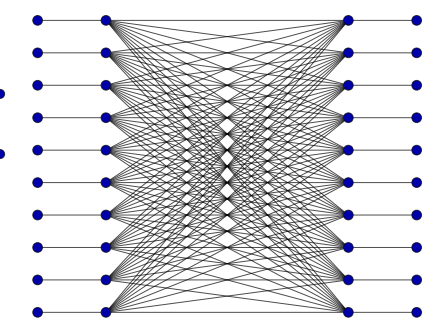

(b)
Figure 2: 20-vertex repeater graphs: (a) a complete graph and (b) a biclique (or complete bipartite graph) with leaves appended to each vertex. In our context,

the graphs underlie all-photonic quantum repeater graph states $\left|R_{C}^{20}\right\rangle$ (from [14]) and $\left|R_{B}^{20}\right\rangle$, respectively.

The vertices correspond to qubits, while the edges represent entanglement localizable through sequences of single-qubit measurements.

\section{PRELIMINARIES}

In this section, we provide the necessary preliminaries to understand our results. We describe the stabilizer formalism and then enumerate some known results on the LU-LC question, which we use as a foundation for the conclusions in Section III.

\section{A. Stabilizer formalism and Minimal Support Condition}

The $n$-qubit Pauli group, $\mathcal{P}_{n}$, consists of operators of the form $P_{1} \otimes \cdots \otimes P_{n}$, where the $P_{i}$ s are either the Pauli matrices or the $2 \times 2$ identity matrix, and we allow a phase of \pm 1 or $\pm i$. A stabilizer is a commutative subgroup of $\mathcal{P}_{n}$ so that $-I$ is not an element of the group. A stabilizer state is the unique eigenstate (with eigenvalue 1) of its stabilizer [24. A graph state, $|G\rangle$, can be defined equivalently as the state stabilized by the set of observables $\left\{K_{a} \mid a \in V_{G}\right\}$, where

$$
K_{a} \equiv X^{a} \bigotimes_{b \in N_{a}} Z^{N_{a}}
$$

and $N_{a}$ denotes the neighbourhood of $a$ : the set of vertices connected (adjacent) to $a$, not including $a$ itself [3]. The stabilizer element, $K_{\ell}$, corresponding to a leaf, $\ell$ (a vertex attached to only a single parent, $p$, as in Fig. 22 is therefore $X^{\ell} Z^{p}$. Stabilizer generators can be conveniently represented as linearly independent rows of an $n \times 2 n$ binary check matrix $S=[X \mid Z]$ where the left (right) sides have 1 s to indicate the indices of $X(Z)$ operators, and 0 otherwise. The check matrix of a graph state, $|G\rangle$, is then $S_{G}=[I \mid \Gamma]$, where $\Gamma$ is the graph's adjacency matrix, that is

$$
\Gamma_{i j}= \begin{cases}0 & \{i, j\} \notin E_{G} \\ 1 & \{i, j\} \in E_{G}\end{cases}
$$

In the discussion that follows, we will refer to the Minimal Support Condition (MSC) 25], a statement about the structure of a stabilizer. We therefore list some definitions important for understanding the condition, using a leaf qubit, $\ell$, for illustration:

- The weight of a stabilizer element is the number of non-identity operators comprising it. The weight of $K_{\ell}$ is 2 .

- The distance of a stabilizer state is the weight of the lowest-weight element in its stabilizer. Any connected graph state with leaves is therefore a distance-2 state.

- The support, $\omega_{a}$, of a stabilizer element is the set of indices of its non-identity elements. For example, $\omega_{\ell}=\{\ell, p\}$. The support is called minimal if there is no other stabilizer element $K_{b}$ with support $\omega_{b}$ so that $\omega_{b} \subset \omega_{a}$. A minimal element is a stabilizer element with minimal support. For any connected graph state with leaves, then, $\omega_{\ell}$ is automatically a minimal support, and $K_{\ell}$ a minimal element.

Definition 1 (Minimal Support Condition [25]). Let $|G\rangle$ be a fully connected graph state with stabilizer $\left\langle K_{a}\right\rangle$. Let $\mathcal{M}$ denote the subgroup of $\left\langle K_{a}\right\rangle$ generated by its minimal elements. Then $|G\rangle$ satisfies the Minimal Support Condition $(M S C)$ iff the Pauli operators $X, Y$ and $Z$ each occur on every qubit in $\mathcal{M}$.

States satisfying the MSC have a rich stabilizer structure with repercussions for their $\mathrm{LU} \Leftrightarrow \mathrm{LC}$ status, as shown in the following section (see also [25]).

\section{B. Review of results on locally equivalent graph states}

Let $|G\rangle$ and $|\tilde{G}\rangle$ be two $n$-qubit graph states. If there exists a local unitary, $U=\bigotimes_{i=1}^{n} U_{i}$, so that $U|G\rangle=|\tilde{G}\rangle$, then the graph states are said to be $L U$-equivalent. If there exists a local Clifford unitary, $U_{C}=\bigotimes_{i=1}^{n} U_{C i}$, so that $U_{C}|G\rangle=|\tilde{G}\rangle$, the graph states are $L C$-equivalent. Local Clifford unitaries are defined through their effect on the Pauli group: $A \in \mathcal{P}_{n} \Longrightarrow U_{C} A U_{C}^{\dagger} \in \mathcal{P}_{n}$ [24].

One important repercussion of the LC-equivalence of two graph states is that their underlying graphs can be transformed between each other with a simple graphical prescription known as the LC Rule, expressed in the following theorem: 
Theorem (LC Rule [3, 17]). Two graph states, $|G\rangle$ and $|\tilde{G}\rangle$, are $L C$-equivalent iff the graphs $G$ and $\tilde{G}$ are related through a sequence of local complementations. The $L C$ unitary that effects a local complementation about vertex $a$ is given by

$$
U_{a}^{L C}(G)=e^{-i \frac{\pi}{4} X^{a}} e^{i \frac{\pi}{4} Z^{N_{a}}} \propto \sqrt{K_{a}} .
$$

Proof. Given in [3, 17].

While there exist graph states for which local Clifford equivalence is not sufficient to describe local unitary equivalence more broadly [21, 22, $\mathrm{LU} \Leftrightarrow \mathrm{LC}$ holds for a large assortment of graph states. We can immediately spot some of these states with a simple fact:

Fact 1. If $L U \Leftrightarrow L C$ holds for a graph state, $|G\rangle$, then it holds for any graph state, $|\tilde{G}\rangle, L C$-equivalent to $|G\rangle$.

Proof. Suppose that $|G\rangle$ satisfies LU $\Leftrightarrow$ LC. Since $|G\rangle$ and $|\tilde{G}\rangle$ are LC-equivalent, we may find a local Clifford unitary, $U_{C}$, so that $|G\rangle=U_{C}|\tilde{G}\rangle$. Let $U$ be an arbitrary local unitary, and suppose there is a graph, $H$, so that $U|\tilde{G}\rangle=|H\rangle$. Then

$$
\begin{aligned}
U|\tilde{G}\rangle=|H\rangle & \Longrightarrow U\left(U_{C}^{-1}|G\rangle\right)=|H\rangle \\
& \Longrightarrow\left(U U_{C}^{-1}\right)|G\rangle=|H\rangle .
\end{aligned}
$$

As $\mathrm{LU} \Leftrightarrow \mathrm{LC}$ holds for $|G\rangle$ and $U U_{C}^{-1}$ is a local unitary, it is true that there exists a local Clifford unitary, $V_{C}$, with

$$
V_{C}|G\rangle=|H\rangle \Longrightarrow V_{C} U_{C}|\tilde{G}\rangle=|H\rangle .
$$

Since the product of two local Clifford unitaries is again a local Clifford unitary, the result follows.

Because any stabilizer state is LC-equivalent to a graph state, Fact 1 implies that answering a question related to the local equivalence of graph states has immediate repercussions for stabilizer states [19, 26, 27].

The following is a sample of six significant Results in the literature that we refer to in Section III] It is known that $\mathrm{LU} \Leftrightarrow \mathrm{LC}$ holds for $|G\rangle$ if

1. $G$ has 8 vertices or fewer [3, 28].

2. $|G\rangle$ is the $n$-Greenberger-Horne-Zeilinger (GHZ) state: $\left|\mathrm{GHZ}_{n}\right\rangle \equiv \frac{1}{\sqrt{2}}\left(|0\rangle^{\otimes n}+|1\rangle^{\otimes n}\right)[25,29]$.

3. $|G\rangle$ satisfies the Minimal Support Condition given in Def. (1) 25.

4. The state obtained by removing the leaves from $G$ satisfies the MSC [15].
5. $G$ has no cycles of length 3 or 4 . (Here a cycle denotes any path that starts and ends at the same vertex, with no edges repeated, and the length is the number of edges along this path.) [15].

6. The stabilizer of $|G\rangle$ has rank of support less than 6 [21].

Armed with the tools from this section, we are ready to investigate LU-LC equivalence in all-photonic quantum repeater graph states.

\section{RESULTS}

\section{A. Complete graph, biclique and repeater graph states}

We begin this section by showing that $n$-GHZ states satisfy LU $\Leftrightarrow$ LC. Although this result was shown in [25], it is instructive to present it as a simple corollary to the results in Section II to contrast the direct approach taken by Van den Nest et al.:

Corollary 1. $n-G H Z$ states satisfy $L U \Leftrightarrow L C$.

Proof. $\left|\mathrm{GHZ}_{n}\right\rangle$ is LC-equivalent to an $n$-qubit star graph state $\left|S_{n}\right\rangle$ (Fig. 3a). To see this, apply prescription (1) to generate $\left|S_{n}\right\rangle$ :

$$
\begin{aligned}
\left|S_{n}\right\rangle & =\mathrm{CZ}^{12} \mathrm{CZ}^{13} \ldots \mathrm{CZ}^{1 n}|+\rangle^{\otimes n} \\
& =|0\rangle|+\rangle^{\otimes n-1}+|1\rangle|-\rangle^{\otimes n-1},
\end{aligned}
$$

where $|-\rangle \equiv \frac{1}{\sqrt{2}}(|0\rangle-|1\rangle)$. Then, applying the gate

$$
\mathcal{H} \equiv I^{1} \otimes H^{2} \otimes \cdots \otimes H^{n}
$$

to $\left|S_{n}\right\rangle$, where the $H^{i}=\frac{1}{\sqrt{2}}\left[\begin{array}{cc}1 & -1 \\ 1 & 1\end{array}\right]$ are Hadamard gates, transforms the state to $\left|\mathrm{GHZ}_{n}\right\rangle$. Since Hadamard gates are local Clifford unitaries, so is $\mathcal{H}$. But star graphs have no cycles of length three or four, implying, by Result 5 . that they satisfy $\mathrm{LU} \Leftrightarrow \mathrm{LC}$. Finally, Fact 1 implies that $\left|\mathrm{GHZ}_{n}\right\rangle$ also satisfies LU $\Leftrightarrow \mathrm{LC}$.

The next result follows immediately:

Corollary 2. Complete graph states satisfy $L U \Leftrightarrow L C$.

Proof. Applying a local complementation to the central qubit of the star graph, $S_{n}$, produces a complete graph, $C_{n}$ (Fig. 3b). By the LC Rule, this implies that $\left|S_{n}\right\rangle$ and $\left|C_{n}\right\rangle$ are LC-equivalent, and the result follows by Fact 1

Somewhat less obviously, it is also true that $\mathrm{LU} \Leftrightarrow \mathrm{LC}$ holds for complete bipartite graph state, or bicliques, as defined and illustrated in Fig. 4b.

Claim 1. Biclique states satisfy $L U \Leftrightarrow L C$. 
Proof. We may not use Result 5 right away: although no bipartite graph can have a cycle of length 3, bicliques have numerous length- 4 cycles. But Fig. 5 shows how three applications of the LC Rule transform the biclique to a "binary star" state (Fig. 4a). Since the binary star has no cycles of length 3 or 4 , Fact 1 and Result 5 now give the result.

Actually, the procedure in the proof to Claim 1 is easily generalized to account for asymmetrical bicliques with a different number of vertices in the left and right sets. See Fig. 6. In what follows, we will use "biclique" to mean "generalized biclique," as this is the convention in the literature.

In proving the previous claim, we presented an $L C$ orbit: the set of states LC-equivalent to a biclique state. Actually, considering (i) the symmetry of the problem; (ii) the fact a local complementation at a leaf vertex has no effect on the graph; and (iii) the fact that the LC operation is a self-inverse, we have provided the complete LC orbit up to the permutation of vertices. In general, although it is easy to identify whether two given graph states are LC-equivalent [18, 19], it is not known whether the LC orbit of an arbitrary graph state can be generated efficiently [3].

To move from the bare complete graph and biclique states to the repeater graph states, it is tempting to use Results 3 or 4 . However, none of the graphs we have discussed satisfy the MSC:

Claim 2. Complete graph, biclique, and repeater graph states do not satisfy the Minimal Support Condition.

Proof. It was shown in [15 that distance-2 stabilizer states, to which graph states with leaves belong, do not satisfy the MSC. Therefore repeater graph states, along with star (binary star) graph states do not satisfy the MSC. But, from [25, 30], we know that the function $A_{\omega}(|\psi\rangle)$ that gives the number of elements in the stabilizer of $|\psi\rangle$ with support $\omega$ is invariant under local unitaries. Therefore the distance of a graph state is LUinvariant, and so all states LC-equivalent to the star (binary star) graph state, namely the complete graph (biclique) state, are distance-two. Thus the complete graph (biclique) states also do not satisfy the MSC.

Alternatively, we know that complete graph states are locally equivalent to $n$-GHZ states, which do not satisfy the MSC, as shown directly in [25].

The next approach is to append a leaf to each vertex in the complete graph and biclique state, and then run the local complementation procedures in Fig. 3 and 5 backwards. This gives us the following result:

Claim 3. Complete-graph-based repeater graph states with one leaf missing and biclique-based repeater graph states with two leaves missing satisfy $L U \Leftrightarrow L C$.

Proof. Follow the LC procedure in Fig. 3 and 5 but with the states in Fig. 7.

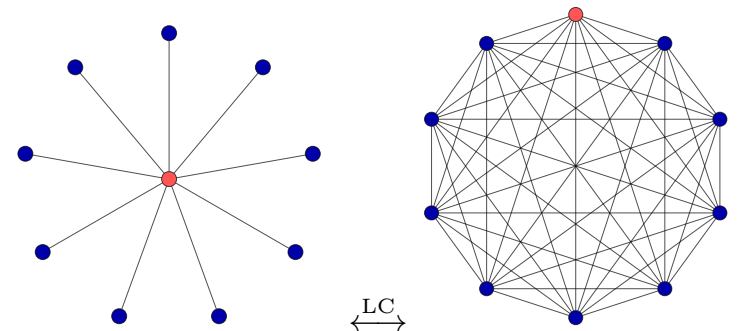

(a) A star graph state,

$\left|S^{n}\right\rangle$, where $n=10$. A

central qubit is connected

to $n-1$ leaf qubits.

(b) A complete graph state, $\left|C^{n}\right\rangle$, where $n=10$.

Every qubit is connected to every other qubit.

Figure 3: Stars and complete graphs are locally equivalent. Local complementation at the red (lighter) vertex on each graph produces the other graph.

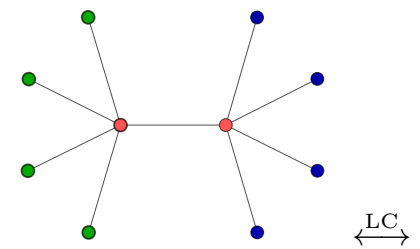

(a) A "binary star" state, $\left|\Sigma^{2 m}\right\rangle$, where $m=5$.

There are two central qubits, each connected to $m-1$ leaves.

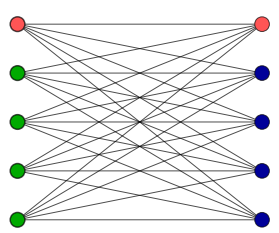

(b) A biclique (or complete bipartite graph) state, $\left|B^{2 m}\right\rangle$, where $m=5$. Complete means each of the $m$ qubits on the left is connected to each of the $m$ qubits on the right; bipartite means the qubits within each set are disconnected.

Figure 4: Binary stars and bicliques are locally equivalent. Local complementation at the central vertices. More detailed procedure in Fig. 5.

It should be noted that running the LC procedure on the perfect repeater graph state will not work: the leaf qubit will end up adjacent to all the neighbours of its parent, introducing cycles of lengths that preclude the use of Result 5. A different strategy is thus needed to verify if LU $\Leftrightarrow$ LC for ideal repeater graph states. For now, this question remains open.

\section{B. More on bicliques}

Although complete graphs were introduced in [14] as the core of the all-photonic quantum repeater, bicliques may freely be used, as the protocol does not require connections among the left and among the right halves of the states. This was noticed independently in [31. As a result, a biclique of $n$ vertices does away with $n(n-2) / 4$ edges from the complete graph without affecting the func- 


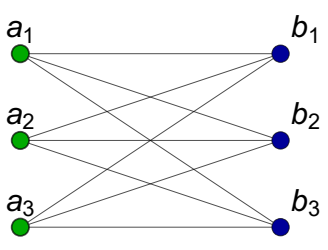

(a)

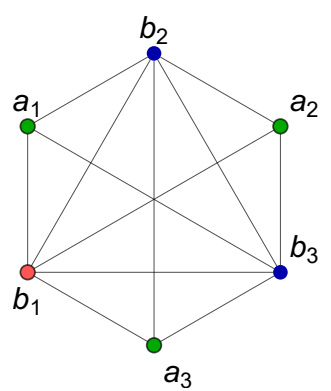

(c)

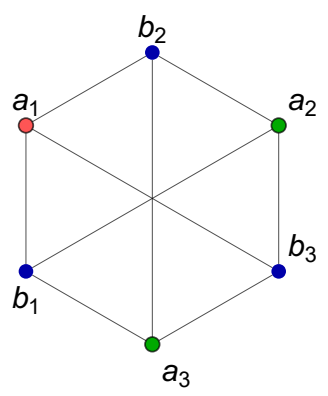

(b)

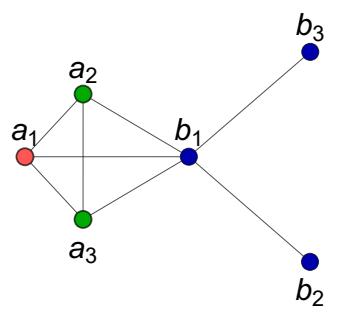

(d)

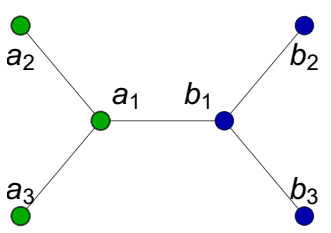

(e)

Figure 5: Procedure for transforming biclique state, $\left|B^{2 m}\right\rangle$, into a "binary star" state, $\left|\Sigma^{2 m}\right\rangle$, through successive applications of the LC Rule. Here $m=3$ but the procedure is general. The nodes on the left half are labelled $a_{i}$ while the nodes on the right half are labelled $b_{i}$. First, unravel the state in (a) to get (b). Then, locally complement the graph in (b) at $a_{1}$ to obtain (c), in which the $b_{i}$ form a complete graph. Next, locally complement the graph in (c) at $b_{1}$ to get a complete graph among $b_{1}$ an all the $a_{i}$. Here $b_{2}, \ldots, b_{m}$ are connected to $b_{1}$ but not to each other. Finally, locally complement the graph in (d) at $a_{1}$ to obtain (e).

tionality of the repeater. A reduction in edges does not imply a reduction in complexity, however; after all, the complete graph, with $n(n+1) / 2$ edges, has only one other state in its LC orbit (up to a permutation of vertices), a simple star graph with $n-1$ edges. But an $n$ vertex biclique is locally equivalent a "binary star," which we named as such because it resembles the fusion of two half-sized stars of $n / 2$ vertices. These states too have $n-1$ edges, offering a comparable complexity for possible advantages.

For one, there might be an experimental graph state generation protocol for which fewer resources are required

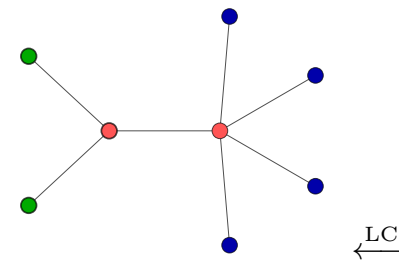

(a) A generalized binary star state, $\left|\bar{\Sigma}^{m, n}\right\rangle$, where $m=3$ and $n=5$. This is the state in 4 a but each of the two central qubits is connected to a different number of leaves.

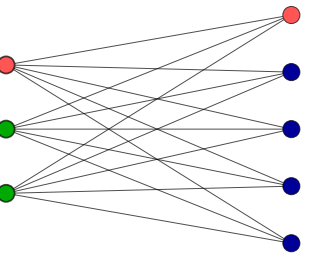

(b) A generalized biclique state, $\left|\bar{B}^{m, n}\right\rangle$, where $m=3$ and $n=5$. This is the state in $4 \mathrm{~b}$ but each of the halves has a different number of qubits.
Figure 6: Generalized binary stars and bicliques are locally equivalent. The steps in Fig. 5 hold but the $(m+n)$-gons in steps (b) and (c) will have some missing sides.

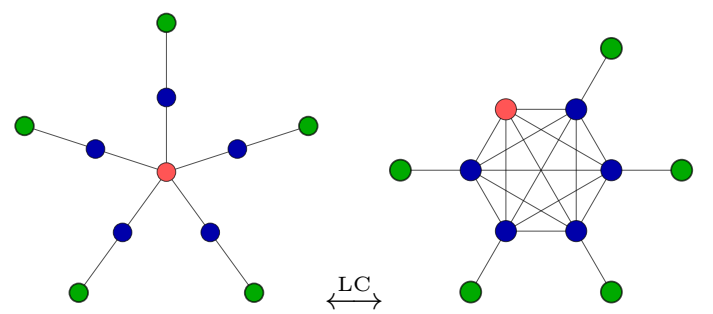

(a)

(b)

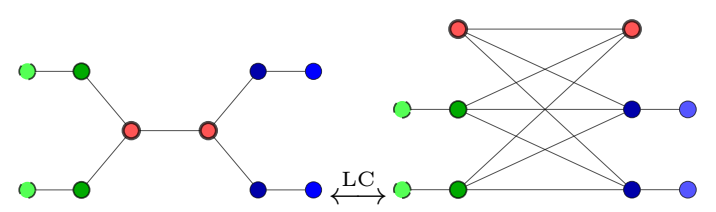

(c)

(d)

Figure 7: Imperfect repeater graph states $(b, d)$ and their LC-equivalent graph states $(\mathrm{a}, \mathrm{c})$. Local complementations at the red (thicker edge) vertices. The more detailed transformation from $\mathrm{c}$ to $\mathrm{d}$ follows the steps in Fig. 5.

to implement the fusion of two stars rather than the creation of one large star. Such a fusion operation exists for photons, for example [5], and photonic repeater graph states can be created through linear optics [4, 14, 32, 33. or through deterministic emitters [34-37]. The discussion in the previous paragraph is relevant to protocols involving multiple solid-state emitters, as the number of edges in a graph state is related to the number of $\mathrm{CZ}$ gates required to couple the quantum dots [31, 36].

Another small benefit of the biclique is in the degree of its entanglement. Two functions quantifying entanglement for graph states are [1, 3, 38]:

- Pauli persistency: $P P(|G\rangle)$, the minimal number of local Pauli measurements required to completely 
disentangle $|G\rangle$.

- Schmidt measure: $E_{S}(|G\rangle)=\log _{2}\left(\min _{|G\rangle} k\right)$, where the minimum is taken over all decompositions

$$
|G\rangle=\sum_{i=1}^{k} \alpha_{i}\left|g_{i}^{1}\right\rangle \otimes \ldots \otimes\left|g_{i}^{n}\right\rangle
$$

for $\alpha_{i} \in \mathbb{C},\left|g_{i}\right\rangle \in \mathbb{C}^{2}$, and $V_{G}=\{1, \ldots, n\}$.

Pauli Persistency is a metric by which cluster states graph states corresponding to lattices in some dimension - were introduced as a powerful resource for quantum computation [1. It provides an upper bound for the Schmidt measure - a proper entanglement monotone and the measures coincide for trees, that is, connected graphs without cycles 3, 38. Furthermore, we need only consider Pauli $Z$ measurements for calculating the Pauli Persistency of tree graph states [3]. Because it takes at least one $Z$ measurement on the central qubit of the star graph state, which is a kind of tree graph, to disentangle it, and because the Pauli Persistency and the Schmidt measure are invariant under local Clifford unitaries, we conclude that $P P=E_{S}=1$ for star graph and complete graph states. Similarly, since at least two $Z$ measurements on the central qubits of the binary star graph state are required disentangle it, $P P=E_{S}=2$ for binary star graph and biclique states. Although these numbers are small - $n$-qubit cluster states have a persistency (and Schmidt measure) of $\left\lfloor\frac{n}{2}\right\rfloor$, for comparison bicliques offer a modest improvement in the robustness of their entanglement over complete graphs. If one star with $n / 2$ qubits (i.e. an $\frac{n}{2}$-GHZ state) is decoupled from the graph, another remains.

Complete bipartite graphs also feature in Rudolph's treatise on silicon-photonic quantum computing [23]. Rudolph does not suggest a biclique as a replacement for the complete graph to underlie the all-photonic quantum repeater, but he describes a crazy graph (Fig. 8a) that can be used for a loss-tolerant encoding scheme. In fact, our result on bipartite graph states applies to crazy graph states of small sizes:

Claim 4. LU $\Leftrightarrow$ LC holds for crazy graph states with two or three columns.

Proof. A crazy graph with two columns is simply a symmetrical bipartite graph. For a crazy graph with three columns, call the vertex sets $L, M$ and $R$ for left, middle and right. Suppose there are $m$ vertices in each column. Notice first that the vertices within $L, M$ and $R$ are disconnected. Notice further that no vertex in $L$ is adjacent to a vertex in $R$, whereas all the vertices in $M$ are adjacent to all the vertices in $L \cup R$. Thus we may rewrite this crazy graph as an asymmetrical biclique state, $\left|\bar{B}^{2 m, m}\right\rangle$ (Fig. 8b), and then use Claim 1 .

If we add more columns to the crazy graph, it still has a bipartite embedding (position all the odd columns as left vertices, and all the even columns as right vertices), but it is no longer a biclique, and so we may not use our results from above. Similarly, the leaves in repeater graphs ensure that the best bipartition is not complete.

We note next that bipartite graphs are the twocolourable graphs, meaning their vertices can be painted in two different colours such that no two vertices of the same colour are adjacent. These states are locally equivalent to Calderbank-Shor-Steane (CSS) states that arise in quantum error correction [12, 39, 40] and feature in schemes for multipartite cryptography [12] and entanglement purification [8]. In fact, Sarvepalli and Raussendorf in 41] show that $\mathrm{LU} \Leftrightarrow \mathrm{LC}$ holds for a certain class of CSS states. However, these CSS states are associated to classical error-correcting codes of distance (and dual distance) of 3 or greater, to which bicliques do not belong, as we now show:

Claim 5. The biclique state corresponds to a CSS code of either distance or dual distance 2. Therefore we may not rely on Corollary 3 in [4] to check if biclique states satisfy $\mathrm{LU} \Leftrightarrow \mathrm{LC}$.

Proof. Consider a biclique of $m$ qubits on the left and $n$ qubits on the right. We can write the check matrix of the stabilizer of this state as follows:

$$
\left[\begin{array}{cc|cc}
I_{m} & 0 & 0 & A \\
0 & I_{n} & A^{T} & 0
\end{array}\right]
$$

where $I_{\alpha}$ is the $\alpha \times \alpha$ identity matrix, $\alpha \in\{m, n\}$, and $A$ is an $m \times n$ matrix consisting only of 1 s. Here we use the convention that the $X(Z)$ operators correspond to the left (right) half of the matrix. Following the argument in Claim 2 of [12] backwards, we may apply the Hadamard matrix to the final $n$ vertices and rearrange the columns to get the new check matrix

$$
\left[\begin{array}{cc|cc}
I_{m} & A & 0 & 0 \\
0 & 0 & A^{T} & I_{n}
\end{array}\right]
$$

But this is a check matrix of a CSS state, since it corresponds to the stabilizer whose elements are products of $X$ and the the identity for the first $m$ qubits, and $Z$ and the identity for the following $n$ qubits [42]. From here, we can read off the parity check matrix for the code, $H(C)=\left[I_{m} \mid A\right]$. But, from [24], we know that the distance of the code is the minimum number of linearly dependent column vectors in the parity check matrix. Since $I_{m}$ always has $m$ linearly independent vectors, and $A$ consists of $n$ columns of 1 s, we conclude that $d=2$ for $n \geq 2$. If $n=1$, we can look at the parity matrix $H\left(C^{\perp}\right)=\left[A^{T} \mid I_{n}\right]$ of the dual code. This matrix has $m+1$ columns of $1 \mathrm{~s}$, meaning the distance of $C^{\perp}-$ the dual distance - is 2 .

\section{Low-rank stabilizer states}

We conclude Section III by clarifying what is meant by Result 6 in Section IIB and whether it applies to our 


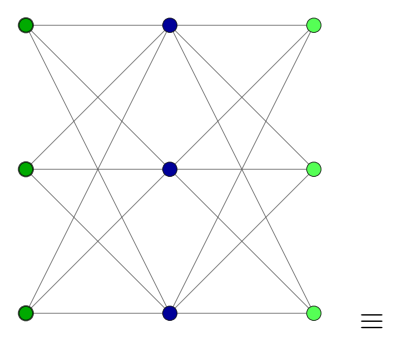

(a) Crazy graph state with three columns and $m=3$ qubits per column.

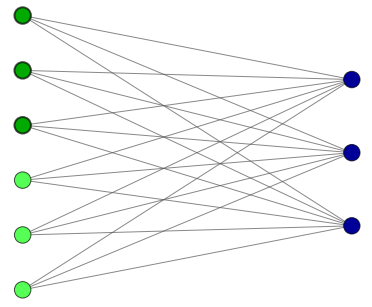

(b) Crazy graph state from (a) with a bipartite embedding. This is an asymmetrical biclique state, $\left|\bar{B}^{2 m, m}\right\rangle$, with $m=3$.

Figure 8: Crazy graph state from [23], used for loss-tolerant encoding.

discussion. In [21], Ji et al. were the first to provide a random search algorithm for generating counterexamples to the LU-LC Conjecture. Their result relies on a reduction of the LU-LC problem to one involving quadratic forms on linear subspaces of $\mathbb{F}_{2}^{n}$, the $n$-bit binary field [43]. In [21, 44, it was shown that any stabilizer state can be written as

$$
|S\rangle=\frac{1}{\sqrt{|S|}} \sum_{x \in S} i^{l(x)}(-1)^{q(x)}|x\rangle,
$$

where $l$ is linear, $q$ is quadratic, and $S$ is a linear subspace of $\mathbb{F}_{2}^{n}$. Ji et al. provide an explicit counterexample in the case $S$ is a rank $r=6$ subspace of $\mathbb{F}_{2}^{27}$, and they prove that no stabilizer state corresponding to a smallerrank subspace can yield counterexamples. To see the implication for graph states, consider the following: A stabilizer state can viewed as a $[[n, 0, d]]$ quantum code [45], and we can interpret $r$ to be the rank of the $X$ part of the check matrix of this code [44], $T=[X \mid Z]$. (Actually, since applying the Hadamard gate to all the qubits swaps the $X$ and the $Z$ parts of $T$, we may also interpret $r$ to be rank of the $Z$ part of the matrix). As in [24, we can perform row reduction on $X$ to obtain

$$
T^{(1)}=\left[\begin{array}{cc|cc}
I_{r} & A & B & C \\
0 & 0 & D & E
\end{array}\right],
$$

where $A$ is an $r \times(n-r)$ matrix, followed by row reduction on $E$ to obtain,

$$
T^{(2)}=\left[\begin{array}{cc|cc}
I_{r} & A & B^{\prime} & 0 \\
0 & 0 & D^{\prime} & I_{n-r}
\end{array}\right] .
$$

Then, applying Hadamard gates to the final $n-r$ qubits, we have

$$
T^{(3)}=\left[\begin{array}{cc|cc}
I_{r} & 0 & B^{\prime} & A \\
0 & I_{n-r} & D^{\prime} & 0
\end{array}\right] .
$$

The stabilizer commutativity condition, $T \Lambda T^{T}=0$, where $\Lambda=\left[\begin{array}{cc}0 & I_{n} \\ I_{n} & 0\end{array}\right]$, implies the right part of $T^{(3)}$ is symmetric, so that $D^{\prime}=A^{T}$ and $B^{\prime}=B^{\prime T}$. Finally, we may set the appropriate qubits of $B^{\prime}$ to 0 , as in [17, to obtain $T^{(4)}=\left[I_{n} \mid \Gamma\right]$, where

$$
\Gamma=\left[\begin{array}{cc}
M & A \\
A^{T} & 0
\end{array}\right] .
$$

Notice that $\Gamma$ is symmetric and has zeros along its diagonal, meaning it is an adjacency matrix of some graph, $G_{S}$. Furthermore, from $T \rightarrow T^{(4)}$ all we have done is apply local Clifford operations and a basis change. This means [17] that the graph state described by the stabilizer matrix $T^{(4)}$ is LC-equivalent to $T$, and hence the corresponding graph state $\left|G_{S}\right\rangle$ is LC-equivalent to $|S\rangle$. When $M \neq 0, \Gamma$ corresponds to a graph with some edges among the qubits $R=\{1, \ldots, r\}$ but none among $L=\{r+1, \ldots, n\}$. When $M=0, \Gamma$ represents a bipartite graph with a bipartition $(L, R)$. For a fixed stabilizer subspace, $S$, the topology of the graph - in other words, the exact form of $A$ and $M$ - depends on the functions $l(x)$ and $q(x)$ in Eq. 5. We can make some general statements, however.

First, let us disambiguate some confusion that exists in physics literature (e.g. [3, 28, 46]) regarding the term "binary rank." For this, we enumerate three kinds of ranks of binary matrices that are used in computer science and mathematics literature [47, 48]. Assume $A$ is an arbitrary $r \times(n-r)$ matrix with entries in $\{0,1\}$, and that $A$ is associated with the upper-right block of the adjacency matrix of a bipartite graph, $G_{A}$, with bipartition $(R, L)$ : i.e., its rows represent the vertices in $R$, and the columns represent the ones in $L$. Without loss of generality, suppose $r \leq n-r$. Then, consider the following definitions:

- Binary rank $\left(\operatorname{rank}_{\mathbb{C}}\right)$ : Normal arithmetic (row reduction), done in quantum information over $\mathbb{C}$. Equal to the biclique partition number, $b p$ : the least number of bicliques needed to partition every edge of $G_{A}$. This means every edge of $G_{A}$ is in exactly one biclique of the partition [47].

- Boolean rank $\left(\operatorname{rank}_{B}\right)$ : Boolean algebra, with addition and multiplication defined through

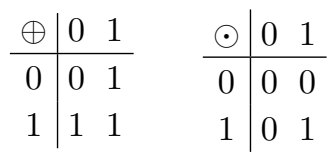

Equal to the biclique cover number, bc: the least number of bicliques needed to cover every edge of $G_{A}$. This means every edge of $G_{A}$ is in at least one biclique of the cover [47].

- XOR rank $\left(\operatorname{rank}_{X}\right)$ : Algebra over $\mathbb{F}_{2}$ (i.e. mod-2 arithmetic), with addition and multiplication defined through [48]:

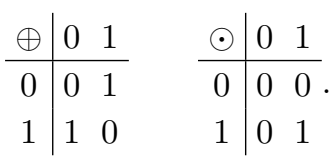


Generally one really means the XOR rank, rather than the binary rank, when dealing with stabilizer operations, since arithmetic is performed modulo 2. Because a set of vectors linearly independent over $\mathbb{C}$ might not be over $\mathbb{F}_{2}$, but the opposite is impossible, we have that

$$
\operatorname{rank}_{\mathrm{X}}(A) \leq \operatorname{rank}_{\mathbb{C}}(A)=b p(A) \leq r .
$$

Hence the XOR rank of $A$ is bounded above by the biclique partition number. For bicliques, we saw in the proof to Claim 5 that $\operatorname{rank}_{X}(A)=1$, and $b p=1$ by definition. This inequality, of course, does not preclude bicliques as counterexamples to LU-LC with the approach in [21]. If we would like to say a little more about the relationship between matrix $A$ and the decomposition (5), consider the following: From [3], we know rank ${ }_{X}$ is equal to the Schmidt rank, $k_{S}^{R}$, of the graph state $\left|G_{A}\right\rangle$ with respect to the bipartition $(R, L)$. According to [46], we may relate the Schmidt rank to $w(|G\rangle)$, the number of minus signs in the computational basis decomposition of the graph state:

$$
k_{S}^{R}=n-\log _{2}\left(2^{n-1}-w\left(\left|G_{A}\right\rangle\right)\right)-1 .
$$

Alternatively, the authors in [3] also show that $k_{S}^{R}=$ $|R|-\operatorname{rank}\left(S_{R}\right)=r-\log _{2}\left|S_{R}\right|$, where $S_{R}$ denotes the subgroup of the stabilizer generated by elements whose support is in $R$. Comparing the expressions, we see that

$$
\left|S_{R}\right|=2^{r}-2^{-(n-r-1)} w\left(\left|G_{A}\right\rangle\right) .
$$

Considering our discussion, then, we have

$$
r-\log _{2}\left|S_{R}\right| \leq b p(A) \leq r,
$$

where $R=\{1, \ldots, r\}$. This means that counterexamples generated by Ji et al.'s procedure, if LC-equivalent to bipartite graphs, will have a biclique partition number between $r-\log _{2}\left|S_{R}\right|$ and $r$. The range of the biclique partition number can then be determined by counting the number of minus signs in the standard basis decomposition. This would require seeing how the negative signs in the coefficients (5) change under the local Clifford gate that takes $|S\rangle$ to a graph state.

Finally, we see that the above observations are consistent with the examples given by Ji et al.: there, the authors provide two $n=27$ qubit graph states. One of the graphs is bipartite, with a bipartition of $r=6$ by $21=n-r$ vertices; the other has an extra edge among the vertices $1, \ldots, 6$. The graphs are proven to be LU equivalent but not LC equivalent. In [22, it's noted that certain sequences of local complementations on $\left|G_{S}\right\rangle$ keep it bipartite and keep the bipartitions the same size, indicating that, perhaps, a necessary condition for counterexamples is $\left|S_{R}\right|=1 \Longrightarrow k_{S}^{R}=r$, and $w\left(\left|G_{S}\right\rangle\right)=2^{n-1}\left(1-2^{-r}\right)$, where $r \geq 6$ is the rank of the stabilizer support.

\section{DISCUSSION}

Although analytical approaches to questions of local equivalence of general graph states are difficult, headway can be made by considering special subsets of graph states. Here we have shown that looking at local Clifford operations only is sufficient to understand the local unitary equivalence of complete graph states, generalized biclique states, imperfect all-photonic quantum repeater graph states and small crazy graphs, as displayed in Figures 3 to 8 . Because LU $\Leftrightarrow$ SLOCC holds for graph states generally [3], this has repercussions for an even broader class of local operations. In turn, for quantum information protocols involving the aforementioned states, one headache of identifying locally equivalent states is relieved.

For bicliques, we have also demonstrated the failure of certain approaches to answer the $\mathrm{LU} \Leftrightarrow \mathrm{LC}$ question: namely, that these states do not satisfy the Minimal Support Condition in [25], are not LC-equivalent to the class of CSS codes described in [41, and do not necessarily correspond to low-rank stabilizer subspaces in [21] that preclude LU-LC counterexamples. We have also provided the LC orbit of a biclique and shown certain advantages in using this state to underlie the all-photonic quantum repeater [14. These boons carry over to other protocols involving a time-reversed entanglement swapping that is the crux of the all-photonic repeater protocol [14].

Though we have demonstrated that bicliques satisfy $\mathrm{LU} \Leftrightarrow \mathrm{LC}$, we saw that the result does not hold for bipartite graphs more generally. The first explicit counterexample to the LU-LC Conjecture in [21] is a 27-qubit state corresponding to a rank-6 stabilizer subspace. We have striven to clarify the relationship between this rank and the properties of the corresponding bipartite graph state, but there is room for further investigation. In [22], Tsimaskuridze and Gühne highlighted the bipartition of Ji et al.'s state and exploited it to construct their own counterexamples. They used graphical rules on non-LC operations aided by so-called hypergraph states, which generalize graph states by allowing an edge to connect to an arbitrary number of vertices. Whether there are counterexamples below 27 qubits, whether there are more efficient and systematic ways of generating them, and whether they have convincing physical interpretations are big open questions in graph state theory that hypergraphs might help to answer.

More humbly, it would be interesting to determine whether $\mathrm{LU} \Leftrightarrow \mathrm{LC}$ holds for perfect repeater graph states based either on complete graphs or a bicliques, and for crazy graph states of an arbitrary number of columns.

\section{ACKNOWLEDGMENTS}

The author would like to thank Hoi-Kwong Lo, Eli Bourassa, Saikat Guha, Aharon Brodutch, and Koji Azuma for helpful discussions. This work is financially supported by the Ontario Graduate Scholarship and the Natural Sciences and Engineering Research Council of Canada (NSERC). 
[1] H. J. Briegel and R. Raussendorf, Phys. Rev. Lett. 86, 910 (2001), arXiv:quant-ph/0004051

[2] M. Hein, J. Eisert, and H. J. Briegel, Phys. Rev. A - At. Mol. Opt. Phys. 69, 062311 (2004), arXiv:quant$\mathrm{ph} / 0307130$

[3] M. Hein, W. Dür, J. Eisert, R. Raussendorf, M. Van den Nest, and H. J. Briegel, (2006), 10.3254/978-1-61499018-5-115, arXiv:quant-ph/0602096.

[4] M. Varnava, D. E. Browne, and T. Rudolph, Phys. Rev. Lett. 97 (2006), 10.1103/PhysRevLett.97.120501, arXiv:quant-ph/0507036

[5] D. E. Browne and T. Rudolph, Phys. Rev. Lett. 95 (2005), 10.1103/PhysRevLett.95.010501, arXiv:quant$\mathrm{ph} / 0405157$.

[6] M. A. Nielsen, Reports Math. Phys. 57, 147 (2006) arXiv:quant-ph/0504097v2.

[7] W. Dür, H. Aschauer, and H.-J. Briegel, Phys. Rev. Lett. 91, 107903 (2003), arXiv:quant-ph/0303087v3

[8] H. Aschauer, W. Dür, and H. J. Briegel, Phys. Rev. A - At. Mol. Opt. Phys. 71 (2005), 10.1103/PhysRevA.71.012319 arXiv:quant-ph/0405045

[9] C. Kruszynska, A. Miyake, H. J. Briegel, and W. Dür, Phys. Rev. A - At. Mol. Opt. Phys. 74 (2006), 10.1103/PhysRevA.74.052316, arXiv:quant-ph/0606090

[10] S. Muralidharan and P. K. Panigrahi, Phys. Rev. A - At. Mol. Opt. Phys. 78 (2008), 10.1103/PhysRevA.78.062333 arXiv:0802.0781 [quant-ph].

[11] A. Keet, B. Fortescue, D. Markham, and B. C. Sanders, Phys. Rev. A - At. Mol. Opt. Phys. 82 (2010), 10.1103/PhysRevA.82.062315, arXiv:1004.4619 |quant$\mathrm{ph}]$

[12] K. Chen and H.-K. Lo, Quantum Inf. Comput. 7, 689 (2004), arXiv:0404133 [quant-ph|.

13] W. Dür, J. Calsamiglia, and H. J. Briegel, Phys. Rev. A - At. Mol. Opt. Phys. 71 (2005), 10.1103/PhysRevA.71.042336 arXiv:quant-ph/0411209v2.

[14] K. Azuma, K. Tamaki, and H.-K. Lo, Nat. Commun. 6, 6787 (2015), arXiv:1309.7207 [quant-ph]

$15 \mid$ B. Zeng, H. Chung, A. W. Cross, and I. L. Chuang, Phys. Rev. A 75, 032325 (2007), arXiv:quant-ph/0611214v2

[16] F. Verstraete, J. Dehaene, B. De Moor, and H. Verschelde, Phys. Rev. A - At. Mol. Opt. Phys. 65, 521121 (2002), arXiv:quant-ph/0109033.

[17] M. V. den Nest, J. Dehaene, and B. De Moor, Phys. Rev. A 69, 022316 (2003), arXiv:quant-ph/0308151

[18] A. Bouchet, Combinatorica 11, 315 (1991) arXiv:cs/0702057v2.

[19] M. Van den Nest, J. Dehaene, and B. De Moor, Phys. Rev. A - At. Mol. Opt. Phys. 70 (2004), 10.1103/PhysRevA.70.034302 arXiv:quant-ph/0405023

[20] O. Krueger and R. F. Werner, arXiv (2005), arXiv:quant-ph/0504166

[21] Z. Ji, J. Chen, Z. Wei, and M. Ying, Quantum Inf. Comput 10, 97 (2007), arXiv:0709.1266.

[22] N. Tsimakuridze and O. Gühne, J. Phys. A Math. Theor 50 (2017), 10.1088/1751-8121/aa67cd, arXiv:1611.06938 [quant-ph].

[23] T. Rudolph, APL Photonics 2, 030901 (2017), arXiv: $1607.08535 \mathrm{v} 1$,

[24] M. A. Nielsen and I. L. Chuang, Cambridge Univ. Press (2010) p. 702.

[25] M. Van den Nest, J. Dehaene, and De Moor, Phys. Rev.
A 71 (2005), 10.1103/PhysRevA.71.062323, arXiv:quant$\mathrm{ph} / 0411115 \mathrm{v} 2$

[26] D. Schlingemann, Quantum Inf. Comput. 2, 307 (2002) arXiv:quant-ph/0111080

[27] M. Grassl, A. Klappenecker, and M. Rötteler, IEEE Int. Symp. Inf. Theory - Proc. , 45 (2002), arXiv:quant$\mathrm{ph} / 0703112$.

[28] A. Cabello, A. J. López-Tarrida, P. Moreno, and J. R. Portillo, Phys. Lett. Sect. A Gen. At. Solid State Phys. 373, 2219 (2009), arXiv:arXiv:0812.4625v3.

[29] D. M. Greenberger, M. A. Horne, and A. Zeilinger, in Bell's Theorem, Quantum Theory and Conceptions of the Universe (Springer Netherlands, Dordrecht, 1989) pp. 69-72, arXiv:0712.0921.

[30] M. Van Den Nest, J. Dehaene, and B. De Moor, Phys. Rev. A - At. Mol. Opt. Phys. 72 (2005), 10.1103/PhysRevA.72.014307, arXiv:quant-ph/0410165.

[31] A. Russo, E. Barnes, and S. E. Economou, arXiv (2018), arXiv:1801.02754

[32] M. Varnava, D. E. Browne, and T. Rudolph, New J. Phys. 9 (2007), 10.1088/1367-2630/9/6/203

[33] M. Varnava, D. E. Browne, and T. Rudolph, Phys. Rev. Lett. 100 (2008), 10.1103/PhysRevLett.100.060502, arXiv:quant-ph/0702044.

[34] N. H. Lindner and T. Rudolph, Phys. Rev. Lett. 103, 1 (2009), arXiv:0810.2587.

[35] S. E. Economou, N. Lindner, and T. Rudolph, Phys. Rev. Lett. 105 (2010), 10.1103/PhysRevLett.105.093601, arXiv:1003.2410

[36] D. Buterakos, E. Barnes, and S. E. Economou, Phys. Rev. X 7 (2017), 10.1103/PhysRevX.7.041023 arXiv:1612.03869

[37] I. Schwartz, D. Cogan, E. R. Schmidgall, Y. Don, L. Gantz, O. Kenneth, N. H. Lindner, and D. Gershoni, Science (80-. ). 49, 1804 (2016), arXiv:1606.07492 [quant$\mathrm{ph}]$

[38] J. Eisert and H. J. Briegel, Phys. Rev. A. At. Mol. Opt. Phys. 64, 1 (2001), arXiv:quant-ph/0007081.

|39| A. R. Calderbank and P. W. Shor, Phys. Rev. A At. Mol. Opt. Phys. 54, 1098 (1996), arXiv:quant$\mathrm{ph} / 9512032$.

[40] A. Steane, Phys. Rev. Lett. 77, 793 (1996)

[41] P. Sarvepalli and R. Raussendorf, Phys. Rev. A - At. Mol. Opt. Phys. 82 (2010), 10.1103/PhysRevA.82.022304 arXiv:0911.1571 [quant-ph].

[42] D. Gottesman, Stabilizer Codes and Quantum Error Correction, Ph.D. thesis (1997), arXiv:quant-ph/9705052

[43] D. Gross and M. Van Den Nest, Quantum Inf. Comput. 8, 263 (2008), arXiv:0707.4000v2

[44] J. Dehaene and B. De Moor, Phys. Rev. A - At. Mol. Opt. Phys. 68, 10 (2003), arXiv:quant-ph/0304125

[45] A. Cross, G. Smith, J. A. Smolin, and B. Zeng, IEEE Trans. Inf. Theory 55, 433 (2009), arXiv:0708.1021v4 [quant-ph].

[46] A. Cosentino and S. Severini, Phys. Rev. A - At. Mol. Opt. Phys. 80 (2009), 10.1103/PhysRevA.80.052309 arXiv:0906.2488v3.

[47] D. A. Gregory, N. J. Pullman, K. F. Jones, and J. R. Lundgren, J. Comb. THEORY, Ser. B 51, 73 (1991).

[48] T. Watson, Chicago J. Theor. Comput. Sci. 1, 1 (2015) 